\title{
Bogolyubov Gaussian Measure in Quantum Statistical Mechanics
}

\author{
D. P. Sankovich
}

Steklov Mathematical Institute of Russian Academy of Sciences, Moscow, Russia

Copyright (C) 2019 by authors, all rights reserved. Authors agree that this article remains permanently open access under the terms of the Creative Commons Attribution License 4.0 International License

\begin{abstract}
The first steps in the application of methods for integrating functions defined on abstract sets were taken by Wiener. Most widely, the ideas of functional integration were developed in Feynman's works. The Feynman continual integral is well known to a wide community of physicists. Along with this, there is another approach to the construction of a functional integral in quantum physics. This approach was proposed by Bogolyubov. Bogolyubov's methods are relevant in quantum statistical physics, and have natural ties with probability theory. We review some mathematical results of integration with respect to a special Gaussian measure that arises in the statistical theory for quantum systems. It is shown that the Gibbs equilibrium averages of the chronological products of Bose operators can be represented as functional integrals with respect to this measure (the Bogolyubov measure). Some properties of this measure are studied. We rewrite partition function of many particle Bose systems in terms of Bogolyubov functional integral.
\end{abstract}

Keywords Bogolyubov Measure, Functional Integral, Gaussian Domination Condition.

\section{Introduction}

This article discusses some work on the space-time or integral over trajectories method in quantum mechanics, considering in particular its application to problems in statistical mechanics and its relation to the theory of a system of interacting bosons.

Evolution of mathematics, by and large, a continuous process and its growth and progress seldom deviate greatly from the natural historical lines. It is because of this that we tend, in retrospect, to admire most those developments which though born well outside it have grown to join and to enrich the mainstream of our science [1]. One of these achievements was the development of a special approach to the study of problems of quantum physics. 65 years ago it was done by N. N. Bogolyubov.

The studies of the problem of integrating functions defined on abstract sets date back to Fréchet's publication [2], where he generalized the Lebesgue method. Somewhat later, these problems were studied by Daniell [3,4], who took advantage of the idea of extending linear functionals. The Daniell theory is based on a family $H(X)$ of elementary functions $h(x)$ on a set $X$ with an elementary integral $I(h)$ defined for them. Under certain conditions, this family can be extended to a broader family $L$ to which the integral $I$ is extended so that $L$ becomes a Banach space with the norm $\|\varphi\|=I(|\varphi|)$. This is the idea of constructing a Lebesgue integral in the Daniell scheme [5,6].

The early results by Wiener [7] have much in common with the theory of the Daniell integral. He defined the integration process for functionals and showed that the integral considered by him is a Daniell integral. Note that in all Wiener's studies starting from 1921, the problem of functional integration is associated with the study of Brownian motion of particles. On the segment $[0,1]$, the set of continuous real functions $C=C[0,1]$ that satisfy the condition $x(0)=0$ is considered. Here, $x(t)$ is the coordinate of a Brownian particle that moves along the axis under the action of random impacts and is located at the origin at $t=0$. The Wiener measure has zero mean and the correlation function $\min (t, s)$. This measure is included in a more general class of measures in function spaces that are called Gaussian measures.

For the first time, the functional integration in quantum physics was used by Feynman [8]. He says in the introduction of paper [9] that this formulation was suggested by remarks of Dirac [10] concerning the relation of the classical action to quantum mechanics. The construction of the Feynman functional (continual) integral shares some common features with the Wiener integral. However, these integrals are essentially different [11]. While the Wiener path integral is based on a well-established mathematical background, the complex oscillatory Feynman and phase-space path integrals still meet some analytical difficulties in rigorous mathematical definition and justification. The Wiener integral is based on a well-defined Gaussian measure, while the Feynman and phase-space path integrals do not admit any strictly defined measure and should be understood as more or less mathematically justified limits of their finite-dimensional approximation.

The idea of expressing physical observables as continual in- 
tegrals was developed in quantum field theory for representing Green functions. Two methods for such a representation appeared almost simultaneously. One of them is based on the formal integration of equations in variational derivatives for Green functions [12-14]. Bogolyubov [15] developed another approach,which was based on the representation of Green functions in terms of vacuum expectations of chronological products; the operation of averaging over a boson vacuum was interpreted as a functional integral. In [16], the Bogolyubov functional integration method was used to study problems of gradient transformations for electrodynamic Green functions in the BlochNordsiek model. Further, Bogolyubov returned to this construction within the framework of statistical mechanics when investigating a polaron model [17]. It was shown in [18] that the measure arising in the Bogolyubov approach is the Gaussian measure in an appropriate space of continuous functions. The Gibbs equilibrium means of the chronological products of operators are expressed as functional integrals with respect to this measure. The quantum-mechanical partition function can be written as an integral over certain imaginary "motions" of the particle. An expressions for the partition functions, reduced density matrices, and correlation functions in terms of functional integrals have been given by many authors [19-25].

The Bogolyubov measure is an important example of Gaussian measures. Other examples are known in the theory of random processes and physics. The most famous are the fractional Brownian motion, the Ornstein-Uhlenbeck process, the Brownian bridge, the Wiener field. A rather large family of examples is given in [26].

This paper does not attempt to be a comprehensive review of the field of Gaussian functional integrals in quantum physics. Our main concern is the review of the Bogolyubov method of functional integration in the problems of equilibrium quantum statistical mechanics.

Below, we consider certain properties of functional integrals with respect to the Bogolyubov measure. In the last decade, experts in probability theory have paid attention to Bogolyubov's measure [27, 30,32,33]. We hope that our brief review will arouse interest in a wider circle of physicists and mathematicians to the questions connected with the application of the Bogolyubov method in various problems of functional analysis, probability theory and physics.

In Section 2 we use the concept of a chronological product ( $T$-product) of operators to derive Bogolyubov functional integral. In Section 3 we calculate certain simple integrals with respect to the Bogolyubov measure and discuss some properties of this measure. In Section 4 we present the results of recent work related to the calculation of the exact asymptotic formulas of some functional integrals with respect to Bogolyubov's measure. In Section 5 we prove an inequality for traces that is used in the theory of phase transitions.

\section{The Bogolyubov Measure}

Bogolyubovs approach to the construction of a functional integral is based on the concept of chronological ordering. The concept of a chronological product ( $T$-product) of operators appeared in quantum mechanics in relation to the Schrödinger equation with a time-dependent Hamiltonian [34]. Such an equation arises in the so-called interaction representation and has the form (hereupon, we assume that the Plank constant divided by $2 \pi$ is equal to unity, $\hbar=1$ )

$$
i \frac{d \Phi(t)}{d t}=\widetilde{H}(t) \Phi(t)
$$

where

$$
\widetilde{H}(t)=e^{i H_{0}\left(t-t_{0}\right)} V e^{-i H_{0}\left(t-t_{0}\right)}
$$

and $H=H_{0}+V$ is a time-independent Hamiltonian of the dynamical system under consideration. If $\Phi$ stands for a timeindependent state vector in the Heisenberg representation, then $\Phi(t)=S\left(t, t_{0}\right) \Phi$, where

$$
S\left(t, t_{0}\right)=e^{i H_{0}\left(t-t_{0}\right)} e^{-i H\left(t-t_{0}\right)}, \quad S\left(t_{0}, t_{0}\right)=I .
$$

The evolution operator $S\left(t, t_{0}\right)$ satisfies the following initial value problem:

$$
i \frac{\partial}{\partial t} S\left(t, t_{0}\right)=\widetilde{H}(t) S\left(t, t_{0}\right), \quad S\left(t_{0}, t_{0}\right)=I .
$$

In quantum mechanics, the operator $S(+\infty,-\infty)$ is called a scattering matrix [35]. The evolution operator $S\left(t, t_{0}\right)$ is a unitary propagator [36], i.e., it satisfies the following conditions:

a) $S\left(t, t_{1}\right) S\left(t_{1}, t_{0}\right)=S\left(t, t_{0}\right)$,

b) $S(t, t)=I$,

c) $S\left(t, t_{0}\right)$ is strongly continuous with respect to the set of variables $t$ and $t_{0}$.

The initial value problem (1) is formally equivalent to the integral equation

$$
S\left(t, t_{0}\right)=I-i \int_{t_{0}}^{t} \widetilde{H}(\tau) S\left(\tau, t_{0}\right) d \tau .
$$

By successive substitutions, we can establish the following Dyson expansion:

$$
S\left(t, t_{0}\right)=\sum_{n=0}^{\infty} S_{n}\left(t, t_{0}\right)
$$

where

$$
\begin{aligned}
S_{n}\left(t, t_{0}\right) & =(-i)^{n} \int_{t_{0}}^{t} d t_{1} \int_{t_{0}}^{t_{1}} d t_{2} \cdots \int_{t_{0}}^{t_{n-1}} d t_{n} \\
& \times \widetilde{H}\left(t_{1}\right) \widetilde{H}\left(t_{2}\right) \ldots \widetilde{H}\left(t_{n}\right) .
\end{aligned}
$$

It is convenient to write relation (3) as

$$
\begin{aligned}
S_{n}\left(t, t_{0}\right) & =\frac{(-i)^{n}}{n !} \int_{t_{0}}^{t} d t_{1} \int_{t_{0}}^{t} d t_{2} \ldots \int_{t_{0}}^{t} d t_{n} \\
& \times T\left[\widetilde{H}\left(t_{1}\right) \widetilde{H}\left(t_{2}\right) \ldots \widetilde{H}\left(t_{n}\right)\right],
\end{aligned}
$$

where we introduce the $T$-product

$$
\begin{aligned}
T\left[V\left(t_{1}\right) V\left(t_{2}\right) \ldots V\left(t_{n}\right)\right] & =\sum \pm \theta\left(t_{i_{1}}>t_{i_{2}}>\ldots>t_{i_{n}}\right) \\
& \times V\left(t_{i_{1}}\right) V\left(t_{i_{2}}\right) \ldots V\left(t_{i_{n}}\right)
\end{aligned}
$$


with

$$
\theta\left(t_{1}>t_{2}>\cdots>t_{n}\right)= \begin{cases}1 & \text { if } t_{1} \geq t_{2} \geq \cdots \geq t_{n}, \\ 0 & \text { otherwise }\end{cases}
$$

The sum in (4) is taken over all possible permutations of indexes $1,2, \ldots, n$. The minus sign corresponds to the Fermi case and is determined by the number of Fermi permutations necessary for obtaining the corresponding term.

Using formula (4), we can rewrite expansion (2) in the following symbolic form:

$$
S\left(t, t_{0}\right)=T \exp \left[-i \int_{t_{0}}^{t} \widetilde{H}(\tau) d \tau\right] .
$$

It follows from the definition of the $T$-product that the operators under the sign of $T$-product commute.

Using the chronological product, we can obtain an important formula of equilibrium statistical mechanics [17]. Consider the operator equation

$$
\frac{d U(s)}{d s}=-\left[H_{0}+H_{1}(s)\right] U(s), \quad U(0)=I .
$$

Here, operator $H_{1}(s)$ may depend on the real parameter $s$. A solution to equation (5) is given by

$$
U(\beta)=T \exp \left\{-\int_{0}^{\beta}\left[H_{0}+H_{1}(\sigma)\right] d \sigma\right\} .
$$

We will assume that $U(s)=e^{-s H_{0}} C(s)$ in (5). Then, the operator $C(s)$ satisfies the initial value problem

$$
\frac{d C(s)}{d s}=-e^{s H_{0}} H_{1}(s) e^{-s H_{0}} C(s), \quad C(0)=I,
$$

which has a solution

$$
C(s)=T \exp \left[-\int_{0}^{s} d \sigma e^{\sigma H_{0}} H_{1}(\sigma) e^{-\sigma H_{0}}\right] .
$$

Therefore,

$$
U(\beta)=e^{-\beta H_{0}} T \exp \left[-\int_{0}^{\beta} d s e^{s H_{0}} H_{1}(s) e^{-s H_{0}}\right] .
$$

Comparing (6) and (7) in the special case when the operator $H_{1}(s)=H_{1}$ is independent of $s$, we obtain the Bogolyubov formula

$$
e^{-\beta\left(H_{0}+H_{1}\right)}=e^{-\beta H_{0}} T \exp \left[-\int_{0}^{\beta} d s e^{s H_{0}} H_{1} e^{-s H_{0}}\right],
$$

where, in the case of interest, $\beta$ is the inverse temperature, $H_{0}$ is the free Hamiltonian, $H_{1}$ is the time-independent Hamiltonian of the interaction. This formula is necessary for representing the partition function in the form of a functional integral.

We recall the Bogolyubov functional integral formalism developed in detail in [18].

Consider a boson of mass $m$ that moves in the space $\mathbb{R}$ and is in the thermodynamic equilibrium with a thermostat at inverse temperature $\beta$. The momentum and coordinate operators, $\hat{p}$ and $\hat{q}$, act in the Hilbert space $L_{2}(\mathbb{R})$ of complex functions $f(x)$ with the inner product $(f, g)=\int_{\mathbb{R}} f(x)=g(x)$ as follows

$$
(\hat{p} f)(x)=-i \frac{d f}{d x}, \quad(\hat{q} f)(x)=x f(x) .
$$

The Bose operators

$$
\hat{b}=\sqrt{\frac{m \omega}{2}} \hat{q}+i \frac{\hat{p}}{\sqrt{2 m \omega}}, \quad \hat{b}^{\dagger}=\sqrt{\frac{m \omega}{2}} \hat{q}-i \frac{\hat{p}}{\sqrt{2 m \omega}}
$$

satisfy the commutation relation $\left[\hat{b}, \hat{b}^{\dagger}\right]=1$.

By

$$
\langle\ldots\rangle_{\widehat{\Gamma}}=\frac{\operatorname{Tr}\left(\ldots e^{-\beta \widehat{\Gamma}}\right)}{\operatorname{Tr} e^{-\beta \widehat{\Gamma}}}
$$

we will denote the Gibbs average with respect to the Hamiltonian $\widehat{\Gamma}$.

Consider the average

$$
\left\langle T \exp \left[i \sum_{k=1}^{N+1} \nu_{k} \widehat{Q}\left(s_{k}\right)\right]\right\rangle,
$$

where $\nu_{k}$ are real numbers and

$$
0=s_{1}<s_{2}<\cdots<s_{k}<\cdots<s_{N}<s_{N+1}=\beta .
$$

The operators $\widehat{Q}(s)$ and $\widehat{\Gamma}$ are defined by the relations

$$
\widehat{Q}(s)=e^{s \widehat{\Gamma}} \hat{q} e^{-s \widehat{\Gamma}}, \quad \widehat{\Gamma}=\frac{\hat{p}^{2}}{2 m}+\frac{m \omega^{2}}{2} \hat{q}^{2},
$$

i.e., we consider a one-dimensional harmonic oscillator. If $\widehat{A}$ is a linear form of Bose operators and $\widehat{\Gamma}$ is a positive definite quadratic Hamiltonian, then the following formula holds [17]:

$$
2 \ln \left\langle e^{\widehat{A}}\right\rangle=\left\langle\widehat{A}^{2}\right\rangle,
$$

Taking into account formula (10), we can write

$$
\begin{aligned}
& \left\langle T \exp \left[i \sum_{k=1}^{N+1} \nu_{k} \widehat{Q}\left(s_{k}\right)\right]\right\rangle \\
& \quad=\exp \left\{-\frac{1}{2} \sum_{n=1}^{N+1} \sum_{m=1}^{N+1} \nu_{n} \nu_{m}\left\langle T\left[\widehat{Q}\left(s_{n}\right) \widehat{Q}\left(s_{m}\right)\right]\right\rangle\right\} .
\end{aligned}
$$

We evaluate the average in the right-hand side of the last relation using the $T$-product definition (4), which leads to the formula

$$
\begin{aligned}
& \left\langle T\left[\widehat{Q}\left(s_{n}\right) \widehat{Q}\left(s_{m}\right)\right]\right\rangle \\
& =\left(2 m \omega\left(1-e^{-\beta \omega}\right)\right)^{-1}\left(e^{-\omega\left|s_{n}-s_{m}\right|}+e^{-\beta \omega+\omega\left|s_{n}-s_{m}\right|}\right) .
\end{aligned}
$$

Thus, the average (8) can be represented as follows:

$$
\begin{aligned}
& \left\langle T \exp \left[i \sum_{k=1}^{N+1} \nu_{k} \widehat{Q}\left(s_{k}\right)\right]\right\rangle \\
& =\exp \left[-\frac{1}{2} \sum_{n, m=1}^{N+1} \nu_{n} \nu_{m}\left(2 m \omega\left(1-e^{-\beta \omega}\right)\right)^{-1}\right. \\
& \left.\quad \times\left(e^{-\omega\left|s_{n}-s_{m}\right|}+e^{-\beta \omega+\omega\left|s_{n}-s_{m}\right|}\right)\right] .
\end{aligned}
$$


Let us write this formula in a form that is more convenient for further analysis. Consider the expression

$$
K\left(s_{n}, s_{m}\right)=e^{-\omega\left|s_{n}-s_{m}\right|}+e^{-\beta \omega+\omega\left|s_{n}-s_{m}\right|},
$$

$0<s_{n}, s_{m}<\beta$, as a function of the variable $s_{n}$. This function, which we denote by $y\left(s_{n}\right)$, satisfies the differential equation

$$
\frac{d^{2} y\left(s_{n}\right)}{d s_{n}{ }^{2}}-\omega^{2} y\left(s_{n}\right)=-2 \omega\left(1-e^{-\beta \omega}\right) \delta\left(s_{n}-s_{m}\right)
$$

and the boundary conditions $y(0)=y(\beta), \quad y^{\prime}(0)=y^{\prime}(\beta)$. We will seek a solution to equation (11) in the form

$$
y(s)=\sum_{n=-\infty}^{\infty} c_{n} e^{2 \pi i n s / \beta} .
$$

We obtain

$$
K\left(s_{j}, s_{k}\right)=2 \omega \frac{1-e^{-\beta \omega}}{\beta} \sum_{n=-\infty}^{\infty} \frac{e^{2 \pi i n\left(s_{j}-s_{k}\right) / \beta}}{\omega^{2}+(2 \pi n / \beta)^{2}} .
$$

Thus, we have the following representation for the average (8):

$$
\left\langle T \exp \left[i \sum_{k=1}^{N+1} \nu_{k} \widehat{Q}\left(s_{k}\right)\right]\right\rangle=e^{-\Omega\left(\left\{\nu_{k}\right\}\right)},
$$

where the form $\Omega\left(\left\{\nu_{k}\right\}\right)$ is quadratic in $\nu_{k}$ and is expressed as

$$
\Omega\left(\left\{\nu_{k}\right\}\right) \equiv \frac{1}{2 m \beta} \sum_{n=-\infty}^{\infty} \frac{\left|\sum_{k=1}^{N+1} \nu_{k} e^{2 \pi i n s_{k} / \beta}\right|^{2}}{\omega^{2}+(2 \pi n / \beta)^{2}} .
$$

It is obvious that $\Omega \geq 0$. Moreover, $\Omega=0$ if and only if $\nu_{1}+\nu_{N+1}=0$ and $\nu_{2}=0, \ldots, \nu_{N}=0$.

Introduce new variables $\eta_{1}=\nu_{1}+\nu_{N+1}, \quad \eta_{2}=\nu_{2}, \ldots$, $\eta_{N}=\nu_{N}$. Then, formula (12) can be rewritten as

$$
\left\langle T \exp \left[i \sum_{k=1}^{N+1} \nu_{k} \widehat{Q}\left(s_{k}\right)\right]\right\rangle=\exp \left(-\frac{1}{2} \sum_{j=1}^{N} \sum_{k=1}^{N} A_{j k} \eta_{j} \eta_{k}\right),
$$

where

$$
\sum_{j, k=1}^{N} A_{j k} \eta_{j} \eta_{k}=\frac{1}{m \beta} \sum_{n=-\infty}^{\infty} \frac{\left|\sum_{k=1}^{N} \eta_{k} e^{2 \pi i n s_{k} / \beta}\right|^{2}}{\omega^{2}+(2 \pi n / \beta)^{2}}
$$

and the covariance matrix has the following elements:

$$
A_{j k}=\frac{1}{2 m \omega \sinh (\beta \omega / 2)} \cosh \left(\frac{\beta \omega}{2}-\frac{\beta \omega}{N}|j-k|\right) .
$$

When deriving this formula, we defined partition (9) by the simple relation $s_{j}=\beta N^{-1}(j-1)$.

Now, let us apply formulas (13) and (14) in order to establish a relation between the Gibbs equilibrium averages of Bose operators and the functional integral.

Consider the expression

$$
\begin{aligned}
\int\left\langle T \exp \left[i \sum_{k=1}^{N+1} \nu_{k} \widehat{Q}\left(s_{k}\right)\right]\right\rangle \\
\quad \times \exp \left\{-i \sum_{k=1}^{N+1} \nu_{k} q_{k}\right\} d \nu_{1} \ldots d \nu_{N} d \nu_{N+1}
\end{aligned}
$$

where $q_{k}$ are real numbers and the integration for each variable $\nu_{i}$ is performed over the entire real axis. Taking into account formula (13) and the well-known values of Gaussian integrals, we obtain

$$
\begin{aligned}
\frac{1}{(2 \pi)^{N+1}} \int\left\langle T \exp \left[i \sum_{k=1}^{N+1} \nu_{k} \widehat{Q}\left(s_{k}\right)\right]\right\rangle \\
\times \exp \left[-i \sum_{k=1}^{N+1} \nu_{k} q_{k}\right] d \nu_{1} \ldots d \nu_{N} d \nu_{N+1} \\
=\rho\left(q_{1}, q_{2}, \ldots, q_{N+1}\right),
\end{aligned}
$$

where

$$
\begin{aligned}
\rho\left(q_{1}, q_{2}, \ldots, q_{N+1}\right) & =\frac{1}{\sqrt{(2 \pi)^{N}}} \frac{\delta\left(q_{1}-q_{N+1}\right)}{\sqrt{\operatorname{det} A}} \\
& \times \exp \left[-\frac{1}{2} \sum_{j, k=1}^{N}\left(A^{-1}\right)_{j k} q_{j} q_{k}\right]
\end{aligned}
$$

$\delta(q)$ is the Dirac delta function, and $A^{-1}$ is the covariance matrix inverse to $A$ with the elements

$$
\left(A^{-1}\right)_{i j}=\frac{m \omega}{\sinh (\beta \omega / N)}\left(2 \cosh \frac{\beta \omega}{N} \delta_{i, j}-\delta_{i, j+1}-\delta_{i, j-1}\right) .
$$

The determinant of the inverse covariance matrix is given by

$$
\frac{(m \omega)^{N}}{2}\left[\left(\cosh \frac{\beta \omega}{N}+1\right)^{N+1}-\left(\cosh \frac{\beta \omega}{N}-1\right)^{N+1}\right] .
$$

It follows from (16) that

$$
\rho \geq 0, \quad \int \rho d q_{1} \ldots d q_{N+1}=1 .
$$

Using (15), we can calculate averages of the form

$$
\left\langle T\left[f\left(\widehat{Q}\left(s_{1}\right), \ldots, \widehat{Q}\left(s_{N+1}\right)\right)\right]\right\rangle .
$$

Indeed, let us take into account the complex Fourier formula

$$
\begin{aligned}
& f\left(Q_{1}, \ldots, Q_{N+1}\right)=\frac{1}{(2 \pi)^{N+1}} \int f\left(q_{1}, \ldots, q_{N+1}\right) \\
& \times \exp \left\{i \sum_{j=1}^{N} \nu_{j}\left(Q_{j}-q_{j}\right)\right\} d q_{1} \ldots d \nu_{N+1}
\end{aligned}
$$

Since the operators $\widehat{Q}\left(s_{j}\right)$ commute under the sign of $T$ product, we have

$$
\begin{aligned}
& \left\langle T\left[f\left(\widehat{Q}\left(s_{1}\right), \ldots, \widehat{Q}\left(s_{N+1}\right)\right)\right]\right\rangle \\
& =\int f\left(q_{1}, \ldots, q_{N+1}\right) \rho\left(q_{1}, \ldots, q_{N+1}\right) d q_{1} \ldots d q_{N+1} .
\end{aligned}
$$

Taking into account properties (17), we see that

$$
\begin{aligned}
0 \leq\left\langle T\left[f\left(\widehat{Q}\left(s_{1}\right), \ldots, \widehat{Q}\left(s_{N+1}\right)\right)\right]\right\rangle & \leq M \\
& \text { if } \quad 0 \leq f\left(\widehat{Q}\left(s_{1}\right), \ldots, \widehat{Q}\left(s_{N+1}\right)\right) \leq M
\end{aligned}
$$


Now, consider functionals $F(q)$ on the real functions ("trajectories") $q(s)$ defined on the segment $0 \leq s \leq \beta$. Let us construct the integral

$$
I \equiv \int F(q) d \mu
$$

with respect to the corresponding measure.

First, we consider a subset of "special functionals" [17] that are continuous functions of a finite number $N$ of variables, $F^{(N)}(q) \equiv \Phi\left(q_{1}, q_{2}, \ldots, q_{N}\right)$, where $q_{j}=q\left(s_{j}\right)$. In this case, we obtain

$I^{(N)}=\int \Phi\left(q_{1}, q_{2}, \ldots, q_{N}\right) \rho\left(q_{1}, q_{2}, \ldots, q_{N}\right) d q_{1} d q_{2} \ldots d q_{N}$

by definition. Then, formulas (18) and (19) imply that

$$
\left\langle T\left[F^{(N)}(\widehat{Q})\right]\right\rangle=\int F^{(N)}(q) d \mu
$$

and that $\left\langle T\left[F^{(N)}(\widehat{Q})\right]\right\rangle \geq 0$ if $F^{(N)}(q) \geq 0$ for arbitrary real numbers $q_{1}, q_{2}, \ldots, q_{N}$. Now, consider a sequence of functions $\left\{q_{N}(s)\right\}, N=1,2, \ldots$, that are defined as follows:

$$
\begin{aligned}
& q_{N}(s)=q\left(s_{j}\right), \quad s_{j} \leq s<s_{j+1}, j=1,2, \ldots, N, \\
& q_{N}(\beta)=q(\beta) .
\end{aligned}
$$

The set of points $\left\{s_{j}\right\}$ is a partition (10) of the segment $[0, \beta]$. We assume that $\left|s_{j+1}-s_{j}\right| \leq \Delta s$ for $j=1,2, \ldots, N$, and suppose that $\Delta s \rightarrow 0$ as $N \rightarrow \infty$. In this case, the sequence of step functions (22) uniformly converges to the function $q(s)$. Path integral (20) can be defined as the limit as $N \rightarrow \infty$ of integrals (21) defined on the subset of special functionals, because the functionals $F\left(q_{N}(s)\right)$ belong to this subset: $I=\lim _{N \rightarrow \infty} I^{(N)}$.

Consider the space $C^{\circ}[0, \beta]$ of continuous functions $q(s)$ defined on the segment $[0, \beta]$ that satisfy the condition $q(0)=$ $q(\beta)$. This is a metric space with respect to the uniform metric $\rho(q, p)=\sup _{s \in[0, \beta]}|q(s)-p(s)|$. The square matrix $A=\left(A_{j k}\right)$ of order $N$ is a positive symmetrical matrix; i.e., the mapping $(j, k) \rightarrow A_{j k}$ is a positive-type kernel on the set $\{1,2, \ldots, N\}$. Hence [37], we can speak of a Gaussian measure $\gamma_{A}$ on the space $\mathbb{R}^{N}$ with covariance $A$. By the Stone-Weierstrass theorem [6], the corresponding set of special functionals is dense in the set of all continuous functions defined on the space $C^{\circ}[0, \beta]$. In the space $C^{\circ}[0, \beta]$, we can introduce a $\sigma$-algebra generated by quasi-intervals (cylindrical sets). This $\sigma$-algebra coincides with the $\sigma$-algebra generated by the sets that are open in the metric $\rho$. The extension of the Gaussian measure from quasi-intervals to their Borel closure determines the Gaussian measure in the space $C^{\circ}[0, \beta][38]$.

\section{Properties of Bogolyubov's Measure}

In Section 2, we showed that in the space $X=C^{\circ}[0, \beta]$ of continuous functions on the segment $[0, \beta]$ that satisfy the condition $x(0)=x(\beta)$ with uniform metric, a Gaussian measure $\mu_{\mathrm{B}}$ is defined with zero average and the correlation function

$$
B(t, s)=\frac{1}{2 m \omega \sinh (\beta \omega / 2)} \cosh \left(\omega|t-s|-\frac{\beta \omega}{2}\right) .
$$

In the measure space $\left\{X, G, \mu_{\mathrm{B}}\right\}$, where $G$ is a distinguished $\sigma$-algebra of subsets in the space $X$, we consider measurable functionals $F(x)$. Then, the following formula holds for the Gibbs equilibrium average of the $T$-product taken with respect to the Hamiltonian $\widehat{\Gamma}$ of the harmonic oscillator:

$$
\langle T[F(\widehat{Q}(t))]\rangle_{\widehat{\Gamma}}=\int_{X} F(x(t)) d \mu_{\mathrm{B}}(x),
$$

where the integral is understood as the Daniell integral over the space $X$. For the integrable functional $F(x)$ the average in formula (24) exists and is finite.

The measure $\mu_{\mathrm{B}}$ thus defined is called the Bogolyubov measure.

Let $a_{1}, a_{2}, \ldots, a_{n}$ be linearly independent elements from a certain separable Hilbert space $H$ whose closure is the support of a measure $\mu$ and that is dense almost everywhere in $X$. Then

$$
\begin{aligned}
\int_{X} F & {\left[\left(a_{1}, x\right),\left(a_{2}, x\right), \ldots,\left(a_{n}, x\right)\right] d \mu(x) } \\
& =(2 \pi)^{-n / 2} \frac{1}{\sqrt{\operatorname{det} A}} \int_{R^{n}} e^{-\left(A^{-1} u, u\right) / 2} F(u) d u
\end{aligned}
$$

if one of these integrals exists, where $A$ is a matrix with the elements $a_{i j}=\left(a_{i}, a_{j}\right)_{H}, i, j=1, \ldots, n, u=\left(u_{1}, \ldots, u_{n}\right)$, and $d u=d u_{1} \cdots d u_{n}$. If we take orthonormalized vectors of the space $H$ as the elements $a_{j}$, then formula (25) reduces to

$$
\begin{aligned}
\int_{X} F\left[\left(a_{1}, x\right),\left(a_{2}, x\right)\right. & \left., \ldots,\left(a_{n}, x\right)\right] d \mu(x) \\
& =(2 \pi)^{-n / 2} \int_{R^{n}} e^{-(u, u) / 2} F(u) d u .
\end{aligned}
$$

Formula (25) has an especially simple form when the functional $F(x)$ depends only on the values of the function $x(t)$ at finite number of points. For example, if $F(x(t))=$ $x\left(t_{1}\right) x\left(t_{2}\right) \ldots x\left(t_{n}\right)$, then the Wick theorem holds, which yields

$$
\begin{aligned}
\int_{X} x\left(t_{1}\right) x & \left(t_{2}\right) \ldots x\left(t_{n}\right) d \mu(x) \\
& =\sum B\left(t_{i_{1}}, t_{i_{2}}\right) B\left(t_{i_{3}}, t_{i_{4}}\right) \ldots B\left(t_{i_{2 k-1}}, t_{i_{2 k}}\right),
\end{aligned}
$$

where $n=2 k$ and the summation is over all $(2 k) ! /\left(2^{k} k !\right)$ ways of partitioning the numbers $1,2, \ldots, 2 k$ into $k$ different unordered pairs, $\left(i_{1}, i_{2}\right),\left(i_{3}, i_{4}\right), \ldots,\left(i_{2 k-1}, i_{2 k}\right)$. This integral vanishes for $n=2 k+1$. In particular, in the case of the Bogolyubov measure,

$$
\begin{aligned}
\left\langle\hat{q}^{2}\right\rangle_{\widehat{\Gamma}} & =\int_{X} x^{2}(t) d \mu_{\mathrm{B}}(x)=B(t, t)=\frac{1}{2 m \omega} \operatorname{coth} \frac{\beta \omega}{2}, \\
\left\langle e^{a \hat{q}^{2}}\right\rangle_{\widehat{\Gamma}} & =\sum_{n=0}^{\infty} \frac{(2 n) !}{2^{n}(n !)^{2}}\left(a\left\langle\hat{q}^{2}\right\rangle_{\widehat{\Gamma}}\right)^{n}=\frac{1}{\sqrt{1-a \operatorname{coth}(\beta \omega / 2) /(m \omega)}},
\end{aligned}
$$

where we should assume that

$$
-m \omega \tanh (\beta \omega / 2) \leq a<m \omega \tanh (\beta \omega / 2)
$$

in the second formula. 
Consider the following quadratic functional on $X$ :

$$
A(x, x)=\sum_{k, j=1}^{\infty} a_{k j}\left(e_{k}, x\right)\left(e_{j}, x\right)
$$

where $a_{k j}=\left(A e_{k}, e_{j}\right)_{H}, A$ is a self-adjoint kernel operator that acts from $H$ to $H$, and $\left\{e_{k}\right\}_{k=1}^{\infty}$ is a basis in $H$. Then, applying formula (25), we can calculate the following integrals:

$$
\begin{aligned}
\int_{X} A(x, x) d \mu(x) & =\operatorname{Tr} A, \\
\int_{X} A^{2}(x, x) d \mu(x) & =(\operatorname{Tr} A)^{2}+2 \sum_{k=1}^{\infty} \lambda_{k}^{2},
\end{aligned}
$$

where $\lambda_{k}$ are the eigenvalues of the operator $A$. The relation

$$
\int_{X} e^{\lambda A(x, x) / 2} d \mu(x)=\frac{1}{\sqrt{D_{A}(\lambda)}}
$$

holds, where $D_{A}(\lambda)$ is the characteristic determinant of the operator $A$ at the point $\lambda, \operatorname{Re} \lambda<\frac{1}{\lambda_{1}}, \lambda_{1}>\lambda_{2}>\ldots$, and

$$
\sqrt{D_{A}(\lambda)}=\sqrt{\left|D_{A}(\lambda)\right|} \exp \left[-\frac{i}{2} \arg D_{A}(\lambda)\right] .
$$

As the quadratic functional in (26), we choose

$$
A(x, x)=\int_{0}^{\beta} x^{2}(t) d t=\sum_{k=-\infty}^{\infty} \lambda_{k}\left(e_{k}, x\right)^{2},
$$

where $e_{k}=\sqrt{\lambda_{k}} \varphi_{k}, \lambda_{k}$ are the eigenvalues of the kernel $B(t, s)$, and $\varphi_{k}$ are the corresponding eigenfunctions. Let us apply the formula [6]

$$
\begin{aligned}
& -\frac{d}{d \lambda} \ln D_{B}(\lambda)=\int_{0}^{\beta} B(t, t) d t \\
& +\lambda \int_{0}^{\beta} B^{(2)}(t, t) d t+\cdots+\lambda^{k} \int_{0}^{\beta} B^{(k)}(t, t) d t+\ldots
\end{aligned}
$$

where $B^{(k)}$ are the corresponding iterated kernels, which are given by

$$
B^{(k)}(t, t)=\frac{1}{\beta m^{k}} \sum_{n=-\infty}^{\infty} \frac{1}{\left[\omega^{2}+\left(2 \pi n \beta^{-1}\right)^{2}\right]^{k}}
$$

in the case of the Bogolyubov measure. Then,

$$
-\frac{d}{d \lambda} \ln D_{B}(\lambda)=\frac{\beta}{2 m} \frac{1}{\sqrt{\omega^{2}-\lambda / m}} \operatorname{coth}\left(\frac{\beta}{2} \sqrt{\omega^{2}-\frac{\lambda}{m}}\right),
$$

where $\lambda<m \omega^{2}$. Integrating this equation, we obtain

$$
D_{B}(\lambda)=\frac{\sinh ^{2}\left(\beta \sqrt{\omega^{2}-\lambda / m} / 2\right)}{\sinh ^{2}(\beta \omega / 2)},
$$

which implies the formula

$$
\begin{aligned}
& \int_{X} \exp \left(\frac{\lambda}{2} \int_{0}^{\beta} x^{2}(t) d t\right) d \mu_{\mathrm{B}}(x) \\
& =\frac{\sinh (\beta \omega / 2)}{\sinh \left(\beta \sqrt{\omega^{2}-\lambda / m} / 2\right)}, \quad \lambda<m \omega^{2} .
\end{aligned}
$$

Note that the moments

$$
m_{k}=\int_{X} A(x, x)^{k} d \mu_{\mathrm{B}}(x)=\int_{X}\left(\int_{0}^{\beta} x^{2}(t) d t\right)^{k} d \mu_{\mathrm{B}}(x)
$$

can be defined by formula (27) and the equality

$$
m_{k+1}=\left.2^{k+1} \frac{d^{k+1}}{d \lambda^{k+1}} \frac{1}{\sqrt{D_{B}(\lambda)}}\right|_{\lambda=0}
$$

In [39] approximate formulas are constructed that are exact for functional polynomials of a given degree, as well as formulas that are exact for integrable functionals of a wider class.

Consider the properties of the support of the Bogolyubov measure in the space $C^{\circ}[0, \beta]$ [40]. It turns out that just as in the case of the Wiener measure, the measure $\mu_{\mathrm{B}}$ is concentrated on continuous but not continuously differentiable paths. Thus, along with the Wiener measure, the Bogolyubov measure provides another important example of everywhere continuous but almost everywhere non differentiable functions.

Introduce the set

$$
C_{h}^{\gamma}\left(t, t^{\prime}\right)=\left\{x \in X:\left|x(t)-x\left(t^{\prime}\right)\right| \leq h\left|t-t^{\prime}\right|^{\gamma}\right\}
$$

where $h>0,0<\gamma \leq 1$, and $t, t^{\prime} \in[0, \beta]$. We seek the Bogolyubov measure of this set. We can write

$$
\mu_{\mathrm{B}}\left\{C_{h}^{\gamma}\left(t, t^{\prime}\right)\right\}=\frac{1}{2 \pi \sqrt{\operatorname{det} K}} \int_{B} d u_{1} d u_{2} e^{-\left(u, K^{-1} u\right) / 2},
$$

where $B=\left\{\left(u_{1}, u_{2}\right) \in R^{2}:\left|u_{1}-u_{2}\right| \leq h\left|t-t^{\prime}\right|^{\gamma}\right\}$. By an appropriate linear change of variables in (28), we obtain

$$
\mu_{\mathrm{B}}\left\{C_{h}^{\gamma}\left(t, t^{\prime}\right)\right\}=\frac{1}{\sqrt{2 \pi}} \int_{-a}^{a} e^{-v^{2} / 2} d v
$$

where

$$
a=\sqrt{\frac{m \omega \sinh (\beta \omega / 2)}{\cosh (\beta \omega / 2)-\cosh \left(\beta \omega / 2-\omega\left|t-t^{\prime}\right|\right)}} h\left|t-t^{\prime}\right|^{\gamma} .
$$

Formula (29) implies an upper estimate for the required measure,

$$
\mu_{\mathrm{B}}\left\{C_{h}^{\gamma}\left(t, t^{\prime}\right)\right\} \leq \sqrt{\frac{2}{\pi}} a .
$$

Now, let us introduce the sets

$$
\begin{aligned}
C_{h}^{\gamma}(t) & =\bigcap_{t^{\prime} \in[0, \beta]} C_{h}^{\gamma}\left(t, t^{\prime}\right)=\left\{x \in X:\left|x(t)-x\left(t^{\prime}\right)\right|\right. \\
& \left.\leq h\left|t-t^{\prime}\right|^{\gamma} \text { for all } t^{\prime} \in[0, \beta]\right\}
\end{aligned}
$$

and

$$
\begin{aligned}
C_{h}^{\gamma} & =\bigcap_{t \in[0, \beta]} C_{h}^{\gamma}(t)=\left\{x \in X:\left|x(t)-x\left(t^{\prime}\right)\right|\right. \\
& \left.\leq h\left|t-t^{\prime}\right|^{\gamma} \text { for all } t, t^{\prime} \in[0, \beta]\right\} .
\end{aligned}
$$

One can prove [1] that the sets $C_{h}^{\gamma}\left(t, t^{\prime}\right), C_{h}^{\gamma}(t)$, and $C_{h}^{\gamma}$ are closed subsets in $X=C^{\circ}[0, \beta]$. 
Consider a sequence $\left\{t_{k}\right\}$ of points from the segment $[0, \beta]$ such that they do not coincide with the point $t$ and $t_{k} \rightarrow t$ as $k \rightarrow \infty$. Then, definition (31), inequality (30), and the downward convexity of the function $\cosh x$ imply that

$$
\begin{aligned}
\mu_{\mathrm{B}}\left\{C_{h}^{\gamma}(t)\right\} & \leq \mu_{\mathrm{B}}\left\{C_{h}^{\gamma}\left(t, t_{k}\right)\right\} \\
& \leq \sqrt{\frac{2 m \sinh (\beta \omega / 2)}{\pi \sinh \left(\beta \omega / 2-\omega\left|t-t_{k}\right|\right)}} h\left|t-t_{k}\right|^{\gamma-1 / 2} .
\end{aligned}
$$

The resulting inequality shows that $\mu_{\mathrm{B}}\left\{C_{h}^{\gamma}(t)\right\}=0$ for $\gamma>$ $1 / 2$; hence,

$$
\mu_{\mathrm{B}}\left\{C_{h}^{\gamma}\right\}=0 .
$$

Recall that a function $x[0, \beta] \mapsto \mathbb{R}$ is said to be Hölder continuous of order $\gamma$ if there is a positive constant $h$ such that $\left|x(t)-x\left(t^{\prime}\right)\right| \leq h\left|t-t^{\prime}\right|^{\gamma}$ for all $t, t^{\prime} \in[0, \beta]$ and $\gamma \in(0,1]$. We have

$$
\Gamma^{\gamma} \equiv\{x \in X: x \text { is a } \gamma \text {-Hölder function }\}=\bigcup_{h=1}^{\infty} C_{h}^{\gamma} .
$$

Hence, by condition (32), the set $\Gamma^{\gamma}$ with $1 / 2<\gamma \leq 1$ is a Borel subset of $X$ with zero Bogolyubov measure (or zero probability). In other words, the Bogolyubov trajectories are not Hölder continuous of order $\gamma>1 / 2$ almost everywhere with respect to the measure. (Consequently, they cannot be continuously differentiable.)

Let $0 \leq t \leq \beta$. We consider the set $D_{t}=\{x \in X$ : $x^{\prime}(t)$ exists $\}$, where $x^{\prime}(t)$ denotes the ordinary derivative of $x$ with respect to $t$ for $t \in(0, \beta)$ and the one-sided derivative for $t=0$ or $t=\beta$. Then, it can be shown [1] that $D_{t} \subset$ $\bigcup_{h=1}^{\infty} C_{h}^{1}(t)$, whence $\mu_{\mathrm{B}}\left(D_{t}\right)=0$.

Define a function $F=X \times[0, \beta] \mapsto \mathbb{R}$ by the relation

$$
F(x, t)=\left\{\begin{array}{l}
1 \text { if } x^{\prime}(t) \text { exists (as a finite function), } \\
0 \text { otherwise. }
\end{array}\right.
$$

It can be shown [1] that the function $F$ is measurable as a function of $x$ and $t$. Then, by the Fubini theorem,

$$
\begin{aligned}
& \int_{X}\left(\int_{0}^{\beta} F(x, t) d t\right) d \mu_{\mathrm{B}}(x) \\
& =\int_{0}^{\beta}\left(\int_{X} F(x, t) d \mu_{\mathrm{B}}(x)\right) d t=\int_{0}^{\beta} \mu_{\mathrm{B}}\left(D_{t}\right) d t=0 .
\end{aligned}
$$

This formula shows that the relation $\int_{0}^{\beta} F(x, t) d t=0$ holds for almost all functions $x$ with respect to the measure $\mu_{\mathrm{B}}$. Consequently, the relation $F(x, t)=0$ holds for almost all functions $x$ with respect to the Bogolyubov measure $\mu_{\mathrm{B}}$ and for almost every $t$ with respect to the Lebesgue measure. Thus, we have proved that the trajectories $x \in X$ are differentiable at most on a subset of $[0, \beta]$ with zero Lebesgue measure.

Since any function $x$ of bounded variation on any interval is differentiable always everywhere on this interval with respect to the Lebesgue measure [41], Bogolyubov trajectories have an unbounded variation on any sub-interval of the segment $[0, \beta]$ with probability 1 .
In [40] the Lévy theorem on the quadratic variation of Wiener trajectories [42] slightly extended to the Bogolyubov measure. A theorem on the quadratic variation of Bogolyubov trajectories is proved. We also study the properties of scale transformations in the Bogolyubov space.

Consider the family of operators $\{T(\beta): 0 \leq \beta<\infty\}$ that act in the space $L^{2}(R)$ by the rule

$$
(T(\beta) f)(x)=\int_{X} d \mu_{\mathrm{B}}(y) f\left(\int_{0}^{\beta} y(t) d t+x\right) .
$$

It is obvious that $T(0)=I$. We obtain

$$
(T(\beta) f)(x)=\sqrt{\frac{m \omega^{2}}{2 \pi \beta}} \int_{-\infty}^{\infty} f(y) \exp \left[-\frac{(y-x)^{2} m \omega^{2}}{2 \beta}\right] d y .
$$

Formula (34) is the well-known formula for the free semigroup in the case of the heat equation. Thus, the family of operators (33) is indeed a strongly continuous semi-group in the space $L^{2}(R)$. The generator of the semi-group is given by

$$
L=\frac{1}{2 m \omega^{2}} \frac{d^{2}}{d x^{2}},
$$

and for any function $f \in L^{2}(R)$, the function $u(\beta, x)=$ $(T(\beta) f)(x)$ is the solution of the Bloch equation

$$
\frac{\partial u}{\partial \beta}=\frac{1}{2 m \omega^{2}} \frac{\partial^{2} u}{\partial x^{2}}
$$

subject to the initial condition $u(0, x)=f(x)$. Formula (33) implies the relation between the Bogolyubov and Wiener measures

$$
\begin{aligned}
\int_{C^{\circ}\left[0, m \omega^{2} t\right]} f\left(x+\int_{0}^{m \omega^{2} t} y(\tau) d \tau\right) d \mu_{\mathrm{B}}(y) & \\
= & \int_{C_{0}^{t}} f(y(t)+x) d \mu_{\mathrm{W}}(y),
\end{aligned}
$$

where $C_{0}^{t}$ is the space of continuous functions on $[0, t]$ that vanish at zero.

A Gaussian random process with a Bogolyubov measure has independent increments, i.e., the random variables $y\left(t_{2}\right)-$ $y\left(t_{1}\right), \ldots, y\left(t_{n}\right)-y\left(t_{n-1}\right)$, where

$$
y(t)=\omega^{-1} x(t)+\int_{0}^{t} x(\tau) d \tau, \quad 0 \leq t \leq \beta,
$$

are independent for any $0<t_{1}<t_{2}<\cdots<t_{n} \leq \beta$.

Gaussian random variable $G \equiv y(t)-y(s)$ is normally distributed with zero average and the variance $(t-s) /\left(m \omega^{2}\right)$, where $t>s$, i.e.,

$$
G \sim N\left(0, \frac{t-s}{m \omega^{2}}\right) .
$$

Note that if $x(t)$ is regarded as a random function, then the integral

$$
\int_{0}^{t} x(\tau) d \tau
$$


introduced above is a stochastic integral defined as the limit in the mean with respect to the given measure for the corresponding integral sums. The integral (36) exists if and only if the mean value $M\left(y^{2}\right)$ exists. This condition is fulfilled for the Bogolyubov measure.

Note that since

$$
\begin{aligned}
\int_{X} d \mu_{\mathrm{B}}(x)\left(\frac{1}{\beta}\right. & \left.\int_{0}^{\beta} x(t) d t\right)^{2} \\
& =\frac{1}{\beta^{2}} \int_{0}^{\beta} d t \int_{0}^{\beta} d \tau B(t-\tau)=\frac{1}{\beta m \omega^{2}},
\end{aligned}
$$

we have

$$
\lim _{\beta \rightarrow \infty} M\left(\frac{1}{\beta} \int_{0}^{\beta} x(t) d t\right)^{2}=0 .
$$

Since a Bogolyubov random process has zero mathematical expectation, $m \equiv M x(t)=0$, we can speak of the ergodicity of this process in the sense that the "time" means ( $\beta$-means) converge in mean square to the "phase" means.

We can establish the connection between Bogolyubov measure and some parabolic partial differential equation [40].

Define a function

$$
\delta_{\beta, \xi}(x)=\frac{1}{2 \pi} \int_{-\infty}^{\infty} d z e^{i z[y(\beta)-y(0)-\xi]},
$$

where $x(t) \in X$ is an arbitrary function, $\xi$ is an arbitrary real number, $\beta$ is a positive number, and $y(t), 0 \leq t \leq \beta$, is defined in (35). Function (37) is an analogue of the Donsker-Lions function [43], which was introduced to investigate the Wiener measure.

Introduce the function

$$
u(\beta, \xi)=E_{\mu_{\mathrm{B}}}\left\{\delta_{\beta, \xi}(x) \exp \left(-\int_{0}^{\beta} V(y(s)-y(0)) d s\right)\right\},
$$

where $V$ is a real function bounded from below. The function (38) is a solution of the partial differential equation

$$
\frac{\partial u}{\partial \beta}=\frac{1}{2 m \omega^{2}} \frac{\partial^{2} u}{\partial \xi^{2}}-V(\xi) u
$$

with the initial condition $u(0, \xi)=\delta(\xi)$ and the boundary conditions $u(\beta, \pm \infty)=0$.

\section{Some Functional Integrals and Mea- sures}

Functional integrals with respect to Gaussian measures can be accurately found only in the simplest cases. Most often one or another asymptotic or approximate calculation is used. In this section, we present the results of recent work related to the calculation of the exact asymptotic formulas of some functional integrals with respect to Bogolyubov's measure. Properties of the Bogoliubov measure, of functional integrals over this measure, and of the Bogolyubov process trajectories were studied in $[27-30,32,33,39,40]$.
Approximate formulas constructed in $[39,44]$ for functional integrals over a Bogolyubov measure are exact for functional polynomials. Some asymptotic formulas for functional integrals with respect to a Bogoliubov measure were obtained in $[28,29,32,33]$, where the asymptotic form of the probability of large deviations of Bogoliubov processes in $L_{p}$-norms, which is important in many problems in probability theory and mathematical physics [45], was also derived. For the probabilities of large deviations of the Bogolyubov measure in the $L^{p}$-norm, the following expressions were obtained in $[29,32]$.

$$
\begin{aligned}
P\left\{\|\xi\|_{p}>\right. & u\} \equiv \mu_{\mathrm{B}}\left\{x: \int_{0}^{\beta}|x(t)|^{p} d t>u^{p}\right\} \\
& =d_{1} u^{-1} \exp \left\{-\frac{m \omega^{2}}{2} \beta^{\frac{2-p}{p}} u^{2}\right\}(1+o(1)),
\end{aligned}
$$

where $0<p<p_{0}, p_{0}=2+4 \pi^{2}(\beta \omega)^{-2}, p \neq 2$ and

$$
d_{1}=\frac{\sqrt{2|p-2|} \beta^{\frac{|p-2|}{2 p}} \sinh \frac{\beta \omega}{2}}{\omega \sqrt{\pi m} \sinh \frac{\beta \omega}{2} \sqrt{|p-2|}} .
$$

For $p=2$, the corresponding estimate is

$$
P\left\{\|\xi\|_{2}>u\right\}=\frac{2 \sqrt{2} \sinh (\beta \omega / 2)}{u \beta \omega^{2} \sqrt{\pi m}} e^{-m \omega^{2} u^{2} / 2}(1+o(1)) .
$$

An interesting feature of the Bogolyubov measure is the occurrence of a threshold value $p_{0}$. This circumstance is due to the Bose character of the systems under study. It is associated with the phenomenon of Bose condensation. For $p=4,6,8,10$ and at the same time $p>p_{0}$ we have

$$
P\left\{\|\xi\|_{p}>u\right\}=d_{2} \exp \left\{-\frac{m \lambda_{0} p}{2 \beta^{\frac{p+2}{p}}} u^{2}\right\}(1+o(1)) .
$$

Constants $d_{2}$ and $\lambda_{0}$ depend only on $p$ and $\beta \omega$. Their specific values are listed in [32]. In the case of the $L^{p}$ functional, that is, a supremum, the following estimates are valid:

$$
\begin{gathered}
\mu_{\mathrm{B}}\left\{x: \sup _{t \in\left[0, \beta_{0}\right]}|x(t)|>u\right\}=2 \beta_{0} \sqrt{\frac{m}{\pi}}\left(\omega \tanh \frac{\beta \omega}{2}\right)^{3 / 2} \\
\times u \exp \left\{-m \omega \tanh \frac{\beta \omega}{2} u^{2}\right\}(1+o(1))
\end{gathered}
$$

$$
\lim _{u \rightarrow \infty} \ln \mu_{\mathrm{B}}\left\{x: \sup _{t \in\left[0, \beta_{0}\right]}|x(t)|>u\right\}=-m \omega \tanh \frac{\beta \omega}{2},
$$

where $0<\beta_{0}<\beta$.

Another fundamental characteristic of random processes (used, e.g., to estimate the accuracy of approximations of random process and to calculate the metric entropy of different sets) is the asymptotic form of their small deviations in one norm or another. In the majority of cases, it is possible to find a rough (logarithmic) asymptotic expression for the probabilities of small deviations for Gaussian processes. Calculating the exact asymptotic form is a much more complicated problem, whose solution is known for only a few processes. 
Pusev [27] evaluates the exact asymptotic form of the probabilities of small deviations for the Bogolyubov process itself and for some related processes with respect to the $L_{2}$-norm. In $[30,31]$ theorems on the exact asymptotic behaviour of small Bogoliubov balls in the $L_{p}$-norm for $2 \leq p \leq \infty$ were obtained.

The problem of small ball behaviour of a random process $X$ in the norm $\|\cdot\|$ is to describe the asymptotics as $\epsilon \rightarrow 0$ of the probability $P\{\|X\| \leq \epsilon\}$. An asymptotic relation of the type

$$
P\{\|X\| \leq \epsilon\} \propto C \epsilon^{\gamma} \exp \left(-d \epsilon^{-\alpha}\right), \epsilon \rightarrow 0
$$

with some real constants $C, \gamma, d, \alpha$ is referred to as exact asymptotics. A less precise statement of the form

$$
\log P\{\|X\| \leq \epsilon\} \propto-d \epsilon^{-\alpha}, \epsilon \rightarrow 0
$$

is known as logarithmic asymptotics.

In [31] the following statement was proved. Let $p>0$ be some fixed number. Then for $\epsilon \rightarrow 0$ there is an asymptotic formula

$$
\begin{aligned}
& P\left\{\int_{0}^{\beta}|x(t)|^{p} d t \leq \epsilon^{p}\right\} \\
& =\epsilon \sqrt{\frac{2 m}{\pi}} \frac{\sinh (\beta \omega / 2)}{\sigma_{p} \beta^{(p+2) / 2 p}} \exp \left(-\frac{\Lambda_{p} \beta^{(p+2) / 2}}{m \epsilon^{2}}\right)(1+o(1)),
\end{aligned}
$$

where

$$
\Lambda_{p}=p 2^{2 / p}\left(\frac{\kappa_{0}}{p+2}\right)^{\frac{p+2}{p}}, \sigma_{p}=\sqrt{\frac{p}{p+2}}\left(\frac{2 \kappa_{0}}{p+2}\right)^{\frac{p+2}{2 p}} .
$$

and $\kappa_{0}$ is a minimal eigenvalue of the self-adjoint Schrdinger operator $\mathcal{S}: L^{2}(\mathbb{R}) \rightarrow L^{2}(\mathbb{R})$,

$$
\mathcal{S} x(t)=-\frac{1}{2} x^{\prime \prime}(t)+|t|^{p} x(t) .
$$

In the particular case of $p=1$ and $p=2$, we get the following result.

$$
\begin{aligned}
& P\left\{\int_{0}^{\beta}|x(t)| d t \leq \epsilon\right\} \\
& =9 \epsilon \sqrt{\frac{m}{2 \pi}} \frac{\sinh (\beta \omega / 2)}{\left(a_{1}^{\prime} \beta\right)^{3 / 2}} \exp \left(-\frac{2\left(a_{1}^{\prime} \beta\right)^{3}}{27 m \epsilon^{2}}\right)(1+o(1)), \\
& P\left\{\int_{0}^{\beta} x^{2}(t) d t \leq \epsilon^{2}\right\} \\
& =4 \epsilon \sqrt{\frac{2 m}{\pi}} \frac{\sinh (\beta \omega / 2)}{\beta} \exp \left(-\frac{\beta^{2}}{8 m \epsilon^{2}}\right)(1+o(1)) .
\end{aligned}
$$

In (40) $a_{1}^{\prime} \approx 1,0188$ is the first zero of the derivative of the Airy function $\mathrm{Ai}^{\prime}(x)$. The relation (41) was first obtained in [27], where the author suggested that $m=\beta=1$.

We now turn to estimates of functional integrals. Formulas for the exact asymptotics of functional integrals with respect to the Bogolyubov measure were obtained in $[28,29,33]$. Functional integrals of two types were considered

$$
\begin{gathered}
I_{1} \equiv \int\left(\int_{0}^{\beta}|x(t)|^{p} d t\right)^{u} \mu_{\mathrm{B}}(x), \\
I_{2} \equiv \int \exp \left[u\left(\int_{0}^{\beta}|x(t)|^{p} d t\right)^{\alpha / p}\right] \mu_{\mathrm{B}}(x),
\end{gathered}
$$

where $0<\alpha<2$ and $p>0$. For integrals of the first type, we have

$$
I_{1}=c_{1} \beta^{u} u^{u p / 2} e^{-q u}(1+o(1)),
$$

where

$$
c_{1}=\frac{\sqrt{2|p-2|} \sinh \frac{\beta \omega}{2}}{\sinh \left(\frac{\beta \omega}{2} \sqrt{|p-2|}\right)}, q=\frac{p}{2}\left(1-\ln \frac{p}{m \beta \omega^{2}}\right)
$$

and $0<p<p_{0},, p \neq 2$. For $p=2$, the corresponding estimate is

$$
I_{1}=\frac{2 \sqrt{2} \sinh \frac{\beta \omega}{2}}{\beta \omega}\left(\frac{2 u}{e m \omega^{2}}\right)^{u}(1+o(1)) .
$$

For $p=4,6,8,10$ and at the same time $p>p_{0}$ we have

$$
I_{1}=c_{2} \sqrt{u}\left(\frac{\beta u}{m}\right)^{u p / 2} \beta^{u} e^{-u p\left(1+\ln \lambda_{0}\right) / 2}(1+o(1)) .
$$

Constant $c_{2}$ depends on $p$ and $\beta \omega$. Its specific value is given in [33].

For integrals of the second type, we have

$$
I_{2}=d_{0} \exp \left[q_{0} u^{\frac{2}{2-\alpha}}\right](1+o(1)),
$$

where $0<p<p_{0}, p \neq 2$ and

$$
\begin{gathered}
d_{0}=\frac{2 \sqrt{|p-2|} \sinh \frac{\beta \omega}{2}}{\sqrt{2-\alpha} \sinh \left(\frac{\beta \omega}{2} \sqrt{|p-2|}\right)}, \\
q_{0}=\frac{2-\alpha}{2}\left(\frac{\alpha}{m \omega^{2}} \beta^{(2-p) / p}\right)^{\alpha /(2-\alpha)} .
\end{gathered}
$$

For $p=2$ we have

$$
I_{2}=\exp \left[\frac{2-\alpha}{2}\left(\frac{\alpha}{m \omega^{2}}\right)^{\frac{2}{2-\alpha}} u^{\frac{2}{2-\alpha}}\right] \frac{4 \sinh \frac{\beta \omega}{2}}{\beta \omega \sqrt{2-\alpha}}(1+o(1)) .
$$

For $p=4,6,8,10$ and at the same time $p>p_{0}$ the following asymptotic formula holds:

$$
I_{2}=K_{1} \exp \left(q_{1} u^{\frac{2}{2-\alpha}}\right) u^{\frac{1}{2-\alpha}}(1+o(1)) .
$$

Specific values of constants $K_{1}$ and $q_{1}$ are listed in [33]. 


\section{Applications for Quantum Statistical Mechanics}

Consider a system with a Hamiltonian $\widehat{H}=\widehat{\Gamma}+\widehat{V}$, where $\widehat{V}=V(\hat{q})$ is an interaction, and consider a one-parameter family of Hamiltonians

$$
\begin{gathered}
\widehat{H}(h)=\widehat{\Gamma}(h)+\widehat{V}, h \in R, \\
\widehat{\Gamma}(h)=\frac{\hat{p}^{2}}{2 m}+\frac{m \omega^{2}}{2}(\hat{q}-h)^{2} .
\end{gathered}
$$

The partition function $Z(h)=\operatorname{Tr} e^{-\beta \widehat{H}(h)}$ of the system takes the following form after the canonical transformation $\hat{q}-h \rightarrow$ $\hat{q}$ :

$$
Z(h)=\operatorname{Tr} e^{-\beta[\widehat{\Gamma}+V(\hat{q}+h)]} .
$$

We will assume that the interaction potential is non-negative and symmetric, i.e., $V(x) \geq 0$ and $V(x)=V(-x)$. Using the operator of chronological ordering, we can write [17]

$$
e^{-\beta(\widehat{\Gamma}+\widehat{V})}=e^{-\beta \widehat{\Gamma}} T \exp \left(-\int_{0}^{\beta} d s e^{s \widehat{\Gamma}} \widehat{V} e^{-s \widehat{\Gamma}}\right) .
$$

Then,

$$
R(h) \equiv \frac{\operatorname{Tr} e^{-\beta \widehat{H}(h)}}{\operatorname{Tr} e^{-\beta \widehat{\Gamma}}}\left\langle T \exp \left[-\int_{0}^{\beta} d s V(\widehat{Q}(s)+h)\right]\right\rangle_{\widehat{\Gamma}} .
$$

Expressing relation (42) via the Bogolyubov functional integral, we obtain

$$
R(h)=\int_{X} \exp \left[-\int_{0}^{\beta} d s V(x(s)+h)\right] d \mu_{\mathrm{B}}(x) .
$$

Now, we apply the theorem on the linear change of variables in the integral with respect to a Gaussian measure [46]. For an integrable functional $F(x)$ and a function $a \in H$, this theorem yields

$$
\int_{X} F(x) d \mu(x)=e^{-\|a\|_{H}^{2} / 2} \int_{X} F(x+a) e^{-(a, x)} d \mu(x) .
$$

let us apply formula (43) in the case of the Bogolyubov measure and constant functions $a$, which in this case belong to $H$. Then,

$$
\begin{aligned}
& \int_{X} F(x+a) d \mu_{\mathrm{B}}(x) \\
= & e^{-\beta m \omega^{2} a^{2} / 2} \int_{X} F(x) \exp \left\{a m \omega^{2} \int_{0}^{\beta} x(t) d t\right\} d \mu_{\mathrm{B}}(x) .
\end{aligned}
$$

Using this relation, we can rewrite the function $R(h)$ as

$$
\begin{aligned}
R(h)=e^{-\beta m \omega^{2} h^{2} / 2} & \int_{X} \exp \left\{-\int_{0}^{\beta} V(x(t)) d t\right\} \\
& \times \exp \left\{m h \omega^{2} \int_{0}^{\beta} x(t) d t\right\} d \mu_{\mathrm{B}}(x) .
\end{aligned}
$$

Now, consider the Fourier-Gauss transform of the functional $f(x)$,

$$
\tilde{f}(y) \equiv F(f ; y)=\int_{X} f(x+i y) d \mu_{\mathrm{B}}(x),
$$

and the Parseval equality

$$
\begin{aligned}
\int_{X} f\left(\frac{x}{\sqrt{2}}\right) & g^{*}\left(\frac{x}{\sqrt{2}}\right) d \mu_{\mathrm{B}}(x) \\
& =\int_{X} F\left(f ; \frac{y}{\sqrt{2}}\right) F^{*}\left(g ; \frac{y}{\sqrt{2}}\right) d \mu_{\mathrm{B}}(y)
\end{aligned}
$$

in the the case of the functionals

$$
f(x)=F(x) \equiv \exp \left\{-\int_{0}^{\beta} d t V(x(t))\right\}
$$

and

$$
g(x)=\exp \left\{m h \omega^{2} \int_{0}^{\beta} x(t) d t\right\} .
$$

Relation (44) takes the form

$$
\begin{aligned}
& \exp \left(\frac{-\beta m h^{2} \omega^{2}}{2}\right) \int_{X} F\left(\frac{x}{\sqrt{2}}\right) \\
& \quad \times \exp \left[\frac{h m \omega^{2}}{\sqrt{2}} \int_{0}^{\beta} x(t) d t\right] d \mu_{\mathrm{B}}(x) \\
& =\int_{X} \widetilde{F}\left(\frac{y}{\sqrt{2}}\right) \exp \left[\frac{i}{\sqrt{2}} h m \omega^{2} \int_{0}^{\beta} y(t) d t\right] d \mu_{\mathrm{B}}(y),
\end{aligned}
$$

which implies that if the inequality

$$
\widetilde{F}(y) \geq 0
$$

holds for any $y$, then

$$
R(h)=\widetilde{F}(-i h) \leq R(0)=\widetilde{F}(0) .
$$

Condition (45) is proved as follows. For symmetric potentials, we have

$$
\begin{aligned}
\widetilde{F}(y) & =e^{(y, y) / 2} \int_{X} \exp \left\{-\int_{0}^{\beta} V(x) d t+i(x, y)\right\} d \mu_{\mathrm{B}}(x) \\
& =e^{(y, y) / 2} \int_{X} \exp \left\{-\int_{0}^{\beta} V(x) d t-i(x, y)\right\} d \mu_{\mathrm{B}}(x) \\
& =\widetilde{F}^{*}(y)
\end{aligned}
$$

i.e., in this case, the Fourier-Gauss transform is real. We prove that it is non-negative. Taking into account that

$$
e^{\|y\|^{2} / 2} \int_{X} e^{i(x, y)} d \mu_{\mathrm{B}}(x)=1,
$$

and applying the Jensen inequality, we obtain

$$
\begin{aligned}
\widetilde{F}(y) & \geq \exp \left\{-e^{\|y\|^{2} / 2} \int_{X} d \mu_{\mathrm{B}}(x) e^{i(x, y)} \int_{0}^{\beta} V(x(t)) d t\right\} \\
& \geq 0
\end{aligned}
$$


which precisely completes the proof of inequality (45).

Condition (46) implies, in particular, that

$$
(\hat{q}, \hat{q})_{\widehat{H}} \leq \frac{1}{\beta m \omega^{2}},
$$

where the Bogolyubov inner product of arbitrary operators $\widehat{A}$ and $\widehat{B}$ is defined as

$$
\begin{aligned}
(\widehat{A}, \widehat{B})_{\widehat{H}} & =(\widehat{B}, \widehat{A})_{\widehat{H}} \\
& =\frac{1}{\beta \operatorname{Tr} e^{-\beta \widehat{H}}} \int_{0}^{\beta} d s \operatorname{Tr}\left[e^{-s \widehat{H}} \widehat{A} e^{-(\beta-s) \widehat{H}} \widehat{B}\right] .
\end{aligned}
$$

If we pass from the operators $\hat{q}$ and $\hat{p}$ to operators $\hat{b}$ and $\hat{b}^{\dagger}$ by the relations

$$
\hat{q}=\frac{1}{\sqrt{2 m \omega}}\left(\hat{b}+\hat{b}^{\dagger}\right), \hat{p}=i \sqrt{\frac{m \omega}{2}}\left(\hat{b}^{\dagger}-\hat{b}\right)
$$

and take into account the selection rules for equilibrium averages with respect to a quadratic Hamiltonian, we can rewrite inequality (47) as

$$
\left(\hat{b}^{\dagger}, \hat{b}\right)_{\widehat{H}} \leq(\beta \omega)^{-1} .
$$

Relation (48) can be used to derive an inequality for the Gibbs equilibrium average $\left\langle\hat{q}^{2}\right\rangle_{\widehat{H}}$. To this end, we should apply the Falk-Bruch inequality [47]. Let

$$
g=\left\langle\hat{q}^{2}\right\rangle_{\widehat{H}}, b=(\hat{q}, \hat{q})_{\widehat{H}}, c=\langle[\hat{q},[\beta \widehat{H}, \hat{q}]]\rangle_{\widehat{H}} \cdot
$$

Suppose that the upper estimates $b \leq b_{0}$ and $c \leq c_{0}$ hold. Then,

$$
g \leq g_{0} \equiv \frac{1}{2} \sqrt{c_{0} b_{0}} \operatorname{coth} \sqrt{\frac{c_{0}}{4 b_{0}}} .
$$

In our case, $b_{0}=\left(\beta m \omega^{2}\right)^{-1}, c_{0}=\beta / m$, and the above inequality yields

$$
\left\langle\hat{q}^{2}\right\rangle_{\widehat{H}} \leq \frac{1}{2 m \omega} \operatorname{coth} \frac{\beta \omega}{2}=\left\langle\hat{q}^{2}\right\rangle_{\widehat{\Gamma}} .
$$

Condition (46) is an example of the so-called Gaussian domination condition [48], and condition (47), which follows from (46), is an example of the so-called local Gaussian domination condition [49], which plays an important role in the theory of phase transitions. An estimate of type (49) was earlier obtained in [50] in a less general case of the one-dimensional non-linear oscillator. Recently, the Gaussian domination was proved for a weakly interacting Bose gas on a cubic lattice [51].

\section{Conclusion}

In this paper we have provided an introductory description of the properties of integration with respect to a special Gaussian measure (the Bogolyubov measure) that arises in the statistical equilibrium theory of quantum systems. As an important application of the general formalism, we have proved the Gaussian domination condition for the non-linear oscillators. From our point of view Bogolyubov's functional integral especially convenient at the decision of the various problems of equilibrium quantum statistical mechanics.

\section{Acknowledgements}

This work is supported by the Program of the Presidium of the Russian Academy of Sciences No.01 "Fundamental Mathematics and its Applications" under grant PRAS-18-01.

\section{REFERENCES}

[1] G. W. Johnson, M. L. Lapidus. The Feynman integral and Feynman's operational calculus, Clarendon, Oxford, 2000.

[2] M. Fréchet. Sur l'intégrale d'une fonctionelle étendue à un ensemble abstrait, Bull. Soc. Math. France, Vol.43, 249-267, 1915 .

[3] P. J. Daniell. A general form of integral, Ann. Math., Vol.19, 279-294,1917-1918.

[4] P. J. Daniell. Integrals in an infinite number of dimensions, Ann. Math., Vol.20, 281-288, 1918-1919.

[5] L. H. Loomis. 1953, An introduction to abstract harmonic analysis, Van Nostrand, Toronto, 1953.

[6] F. Riesz, B. Sz.-Nagy. Functional analysis, Ungar, New York, 1955.

[7] N. Wiener. The mean of a functional of arbitrary elements, Ann. Math., Vol.22, 66-72, 1920.

[8] R. P. Feynman. The principle of least action in quantum mechanics. Ph. D. Dissertation, Physics. Princeton University, Princeton, 1942. (Available from University Microfilms Publication, No. 2948, Ann Arbor, Michigan.)

[9] R. P. Feynman. Space-time approach to non-relativistic quantum mechanics, Rev. Mod. Phys., Vol.20, 367-387, 1948.

[10] P. A. M. Dirac. The Lagrangian in Quantum Mechanics, Phys. Z. Sovjetunion, Vol.3, 64-72, 1933.

[11] R. H. Cameron. A family of integrals serving to connect the Wiener and Feynman integrals, J. Math. and Phys., Vol.39, 126-140, 1960.

[12] S. F. Edwards, R. E. Pierls. Field Equations in functional form, Proc. Roy. Soc., Vol.A224, 24-33, 1954.

[13] I. M. Gelfand, R. A. Minlos. Solution of equations of quantized fields, Dokl. Akad. Nauk SSSR, Vol.97, 209-212, 1954. 
[14] E. S. Fradkin. Green function for interaction between nucleons and mesons, Dokl. Akad. Nauk SSSR, Vol.98, 47-50, 1954.

[15] N. N. Bogolyubov. On the representation of the GreenSchwinger functions in terms of functional integrals, Dokl. Akad. Nauk SSSR, Vol.99, 225-226, 1954.

[16] A. V. Svidzinskii. Calculation of Green functions in the Bloch-Nordsiek model by functional integral method, Zh. Eksp. Teor. Fiz., Vol.31, 324-329, 1956.

[17] N. N. Bogolyubov, N. N. Bogolyubov, Jr. Some aspects of polaron theory, World Scientific Pub., Singapore, 1987.

[18] D. P. Sankovich. Gaussian functional integrals and Gibbs equilibrium averages, Theor. Math. Phys., Vol.119, 345-352, 1999.

[19] R. P. Feynman. Atomic theory of the $\lambda$ transition in helium, Phys. Rev., Vol.91(6), 1291-1301, 1953.

[20] I. M. Gelfand, A. M. Yaglom. Integration in function spaces and its application in quantum physics, J. Math. Phys., Vol.1, 48-69, 1960.

[21] D. ter Haar. Elements of statistical mechanics, Rinehart and Company, Inc., New York, 1954.

[22] S. Albeverio, R. Høegh-Krohn. Uniqueness of the physical vacuum and the Wightman functions in the infinite volume limit for some non polynomial interactions, Commun. Math. Phys., Vol.30, 171-200, 1973.

[23] J. Fröhlich, Y. M. Park. Correlation inequalities and the thermodynamic limit for classical and quantum continuous systems. II Bose-Einstein and Fermi-Dirac statistics, J. Stat. Phys., Vol.23(6), 701-753, 1980.

[24] F. A. Berezin. The method of second quantization, Academic Press, New York, 1966.

[25] V. N. Popov. Functional integrals in quantum field theory and statistical physics, Dordrecht, Reidel, 1983.

[26] S. Watanabe. Stochastic Differential Equations and Malliavin Calculus., Tata Institute of Fundamental Research, SpringerVerlag, Berlin and New York, 1984.

[27] R. Pusev. Asymptotics of small deviations of the Bogoliubov processes with respect to a quadratic norm, Theor. Math. Phys., Vol.165, 1348-1357, 2010.
[28] V. R. Fatalov. Some asymptotic formulas for the Bogoliubov gaussian measure, Theor. Math. Phys., Vol.157, 16061625, 2008 .

[29] V. R. Fatalov. Laplace-type exact asymptotic formulas for the Bogoliubov Gaussian measure, Theor. Math. Phys., Vol.168, 1112-1149, 2011.

[30] V. R. Fatalov. Asymptotic behaviour of small deviations for Bogoliubov Gaussian measure in the $L^{p}$ norm, $2 \leq p \leq \infty$, Theor. Math. Phys., Vol.173, 1720-1733, 2012.

[31] V. R. Fatalov. Gaussian Ornstein-Uhlenbeck and Bogoliubov processes: Asymptotics of small deviations for $L_{p}$-functionals, $0<p<\infty$, Prob. Inform. Transm., Vol.50, 371-389, 2014.

[32] V. R. Fatalov. Exact Laplace-type asymptotic formulas for the Bogolyubov Gaussian measure: The set of minimum points of the action functional, Theor. Math. Phys., Vol.191, 870-885, 2017.

[33] V. R. Fatalov. Functional Integrals for the Bogoliubov Gaussian Measure: Exact Asymptotic Forms, Theor. Math. Phys., Vol.195, 641-657, 2018.

[34] F. J. Dyson. The radiation theories of Tomonaga, Schwinger, and Feynman, Phys. Rev., Vol.75, 486-502, 1949.

[35] N. N. Bogolyubov, D. V. Shirkov. Introduction to the Theory of Quantum Fields, 2nd ed., Wiley, New York, 1980.

[36] M. Reed, B. Simon. Methods of Modern Mathematical Physics, V.2, Acad. Press, New York, 1975.

[37] N. Bourbaki. Éléments de mathématique. Livre VI. Integration, Hermann, Paris, 1952.

[38] Yu. L. Dalecky, S. V. Fomin. Measures and differential equations in infinite-dimensional space, Kluwer, Dordrecht, 1991.

[39] D. P. Sankovich. Some properties of functional integrals with respect to the Bogolyubov measure, Theor. Math. Phys., Vol.126, 121-135, 2001.

[40] D. P. Sankovich. Metric properties of Bogolyubov trajectories in statistical equilibrium theory, Theor. Math. Phys., Vol.127, 513-527, 2001.

[41] H. L. Royden. Real Analysis, 3rd ed., Macmillan, New York, 1988.

[42] P. Lévy. Le mouvement brownien plan, Am. J. Math., Vol.62, 487-550, 1940. 
[43] Hui-Hsiung Kuo. Gaussian measures in the Banach spaces, Springer, Berlin, 1975.

[44] D. P. Sankovich. The Bogolyubov Functional Integral, Proc. Steklov Inst. Math., Vol.251, 213-245, 2005.

[45] H. Touchette. The large deviation approach to statistical mechanics, Phys. Rep., Vol.478, 1-69, 2008.

[46] L. A. Yanovich. Approximate calculation of continual integrals with respect to gaussian measures [in Russian], Nauka i Tekhnika, Minsk, 1976.

[47] H. Falk, L. W. Bruch. Susceptibility and fluctuation, Phys. Rev., Vol.180, 442-444, 1969.

[48] J. Fröhlich. The pure phases (harmonic functions) of generalized processes or: Mathematical physics of phase transitions and symmetry breaking, Bull. Am. Math. Soc., Vol.84, 165-193, 1978.

[49] D. P. Sankovich. Gaussian domination and phase transitions in systems with continuous symmetry, Theor. Math. Phys., Vol.79, 656-665, 1989.

[50] N. N. Bogolyubov, Jr., D. P. Sankovich. Gaussian domination: quantum non-linear oscillator, Phys. Lett. A, Vol.137, 179-182, 1989.

[51] D. P. Sankovich. Proof of Bose condensation for weakly interacting lattice bosons, J. Phys. Commun., Vol.2, 105015, 2018. 